\title{
Ensino da saúde coletiva na Faculdade de Medicina do ABC: alguns apontamentos sobre os desafios da Saúde Coletiva na formação médica
}

\author{
Teaching of collective health in the ABC Medical School: some notes on the \\ challenges of collective health in medical training
}

Nivaldo Carneiro Junior

${ }^{1}$ Faculdade de Medicina do ABC (FMABC) - Santo André (SP), Brasil.

DOI: http://dx.doi.org/10.7322/abcshs.v40i3.820

\begin{abstract}
RESUMO
Introdução: A saúde coletiva, campo de saberes e práticas sobre o processo saúde-doença-cuidado, leva às graduações em saúde questões teórico-metodológicas e tecnologias de intervenções ao exercício profissional. Historicamente, posiciona-se à margem do projeto político-pedagógico da formação em saúde, particularmente médica, de modelo biologizante e hospitalocêntrico. De acordo com a Constituição de 1988, Saúde é um Direito Social e o Sistema de Saúde, público e universal. Surgem formulações às políticas educacionais e saúde. É competência do Sistema Único de Saúde (SUS) ordenar a formação de recursos humanos. As Diretrizes Curriculares Nacionais para as graduações em saúde apontam perfis profissionais voltados às necessidades de saúde da população, exigindo novas metodologias de ensino-aprendizagem e cenários de práticas pedagógicas. Nessa perspectiva, o ensino da saúde coletiva emerge com importância singular. Relato de Experiência: A saúde coletiva na Medicina da Faculdade de Medicina do ABC era grade de disciplinas básicas do $2^{\circ}$ ano. Um novo currículo modifica essa inserção. Até 2013, disciplina anual, em 3 módulos: epidemiologia, bioestatística e políticas e organização de serviços de saúde. Temas de ciências sociais e humanas desenvolviam-se no $1^{\circ}$ ano, módulo interdisciplinar Bases do Exercício Profissional. A grande concentração, nesse período, das disciplinas de morfologia e fisiologia, aproximações incipientes nos serviços de saúde e baixa exposição aos problemas de adoecimento e cuidado da população desafiavam o ensino-aprendizagem da saúde coletiva. Conclusão: Desenvolveram-se estratégias pedagógicas que superassem esse contexto, propiciando experiências cognitivas e habilidades psicomotoras favoráveis à apreensão dos conteúdos da saúde coletiva. Resultados positivos do exame recente do Conselho Regional de Medicina fortalecem essas iniciativas pedagógicas.
\end{abstract}

Palavras-chave: educação médica; saúde pública; currículo; ensino.

\begin{abstract}
Introduction: Collective health, a field of knowledge and practices concerning health, illness and care, introduces medicine students to methodological and theoretical issues and intervention technologies for professional practice. Historically, collective health occupies a subordinate position within the political and pedagogical project which orients health education. This project is hospital-centered and emphasizes biological themes. According to the Brazilian's 1988 Constitution, health is a social right and the Health System is public and universal. New educational and health policies were then formulated. It is the role of Unified Health System to organize the training of human resources. The National Curriculum Guidelines for health undergraduate courses point to professional profiles which can meet population's health needs. This requires new teaching methodologies and scenarios for pedagogical practices. From this view, the collective health teaching becomes very important. Experience report: Collective health in the Medical course at ABC Medical School was one of the basic disciplines for second year students. A new curriculum changed this. Until 2013, it was an annual discipline in three modules: epidemiology, biostatistics and policies and organization of health services. During the first year, themes of social and human sciences were approached as part of Professional Practices Basis. But the teaching of collective health faces several challenges: the great concentration, during this period, of Morphology and Physiology subjects, few contacts with health services and problems concerning illness and care. Conclusion: To solve this, new pedagogical strategies were developed, providing cognitive experiences and skills suitable to learning collective health subject matters. Positive results showed in recent Regional Medical Council examination supports these pedagogical strategies.
\end{abstract}

Keywords: education, medical; public health; Curriculum; teaching. 


\section{INTRODUÇÃO}

A formação profissional constitui um tema importante e sempre atual que é preocupação constante entre educadores, pesquisadores e formuladores de políticas educacionais. Seja qual for a área do conhecimento e da sua aplicabilidade, formar ou, melhor dizendo, disponibilizar profissionais para o mundo do trabalho é uma tarefa nada fácil, que exige sistematização, diálogo com a realidade, incorporação de tecnologias, problematização, entre tantas outras dimensões, que envolvem um complexo campo científico, técnico e social ${ }^{1}$.

Ao tomarmos essas considerações iniciais para a formação do profissional de saúde, essa complexidade se torna exponencialmente maior pelo fato de estarmos lidando com processos e práticas sociais e institucionais externos ao sistema educacional propriamente dito, isto é, vamos ao encontro do universo dos serviços e sistemas de saúde, que tem, entre outras características fortes, uma dimensão política em constante tensão no cotidiano das práticas profissionais e dos modos de vida ${ }^{1,2}$.

O médico e a formação médica se destacam, nesse cenário, pela sua centralidade histórica no saber e no saber-fazer do processo saúde-doença-cuidado, relacionados com as determinações das relações Estado, sociedade e mercado ${ }^{2,3}$.

Nesse sentido, perguntas como "a formação médica tecnicista e centrada no hospital especializado é deformadora?", "que médicos devemos formar?", "os médicos sabem cuidar dos problemas de saúde da população?", "a prática médica se volta para os interesses da população?”, "o modelo de ensino favorece ao futuro médico um entendimento sobre o seu papel social?”, entre outras questões, geram correntes de pensamento e movimentos político-sociais que, de fora e de dentro, articulam-se com problemas político-pedagógicos nos vários movimentos de reforma do ensino médico, que tem tomado corpo doutrinário a partir das primeiras décadas do Século $\mathrm{XX}^{4,5}$.

É nessa perspectiva que se insere a reflexão sobre o ensino da saúde coletiva na formação médica no Brasil, que toma corpo a partir dos finais dos anos 1970, no contexto do movimento da reforma sanitária brasileira, influenciado e influenciando os Departamentos de Medicina Preventiva e Social das Faculdades de Medicina (outras denominações para a área podem ser encontradas, como, por exemplo, Saúde da Coletividade, adotada pela Faculdade de Medicina do ABC - FMABC) e demais grupos de docentes próximos a esse campo ${ }^{6}$.

A saúde coletiva, como campo de saberes e práticas sobre os processos de saúde-doença-cuidado, tem levado à área de educação médica, como também às demais áreas da graduação em saúde, aportes teóricos, metodológicos e tecnologias de intervenções fundamentais para a iniciação e exercício profissional, trazendo para os espaços de ensino-aprendizagens a identificação e problematização da realidade social que determina processos de adoecimento, cuidado e morte da população, através de suas disciplinas fundantes: epidemiologia, ciências sociais e humanas e políticas, gestão, planejamento e avaliação em saúde ${ }^{6}$.

Portanto, a saúde coletiva dirige-se a um outro polo da formação profissional do médico, isto é, trata do aspecto coletivo da saúde, do adoecimento e das formas de cuidado, sejam eles institucionais ou não. Essa perspectiva de abordagem tem sido, historicamente, colocada à margem do projeto político-pedagógico hegemônico da formação médica, muitas vezes em tensão e em disputa com este, repercutindo na estruturação e distribuição da carga horária na grade curricular, nas inserções institucionais, na busca de estratégias educacionais extra-muro da Escola médica, na aceitação dos próprios alunos, entre outros ${ }^{7,8}$.

Com a Constituição brasileira de 1988, na qual a Saúde é um Direito Social e o sistema de saúde é público e universal, novos cenários despontam nas formulações das políticas educacionais e de saúde, especialmente porque é constitucional a competência do Sistema Único de Saúde (SUS) em ordenar a formação de recursos humanos. Isso abre novas possibilidades ${ }^{9}$.

As Diretrizes Curriculares Nacionais (DCN) de 2001 para todas as graduações em saúde resultam desse novo cenário, pois apontam para que os perfis profissionais estejam voltados para o atendimento das necessidades sociais e de saúde da população, repercutindo nas metodologias de ensino-aprendizagem e nos cenários de práticas. Nessa perspectiva, o hospital não é o centro formador e a multidisciplinaridade e a inserção nos serviços e espaços mais capilares da realidade social da população se tornam centrais na organização do projeto político-pedagógico dos cursos de graduação ${ }^{10}$.

Hoje, na Medicina, estamos com a nova DCN, de 2014, que aprofunda e qualifica a anterior, reforçando os eixos da integralidade, gestão da clínica, cuidado, e atenção primária à saúde como fundamentais na estruturação do currículo. Esse cenário coloca a saúde coletiva em posição favorável, não, é certo, de supremacia, mas de cogestora do novo projeto político-pedagógico da formação médica (e de outros cursos da área da saúde também $)^{11}$.

\section{RELATO DE EXPERIÊNCIA}

\section{O ensino da saúde coletiva na FMABC}

As considerações acima contextualizam e também determinam a inserção e o ensino da saúde coletiva no curso de Medicina da FMABC. De fato, é preciso não esquecer as especificidades próprias dessa Instituição de Ensino Superior: privada, de inserção regional e sem serviços hospitalares próprios, entre outras.

A temática da Saúde Coletiva faz parte do Currículo da FMABC desde 1969. A princípio, com apenas 60 horas/aula no ano, era chamada de Medicina Preventiva e ministrada apenas no $1^{\circ}$ ano do curso de Medicina. Sua carga horária cresceu gradativamente e em 1972, com 450 horas/ano, é ministrada nos três primeiros anos de curso. Nesse mesmo ano, chama a atenção a presença do 
conteúdo em Educação Médica, com 120 horas/aula. A partir de 1972, a carga horária da disciplina tem redução, passando a ser denominada de Saúde Coletiva. Em 1979, restringe-se ao $2^{\circ}$ ano do curso médico.

Em 1989, ocorre, na disciplina, um processo de reorganização com os módulos de epidemiologia, bioestatística e políticas e organização de serviços de saúde, sempre no $2^{\circ}$ ano do curso de Medicina, mas com algumas inserções no $1^{\circ}$ ano, inicialmente, como ciências sociais aplicadas à saúde.

Entre 2000 e 2002, desenvolve conteúdos de saúde coletiva no $5^{\circ}$ ano de Medicina, como parte do estágio de clínica médica no Centro de Saúde-Escola de Capuava.

A saúde coletiva no curso de Medicina da FMABC fazia parte, até 2013, da grade curricular das disciplinas básicas, no $2^{\circ}$ ano. Um novo currículo tem início naquele ano e essa distribuição é alterada. Nesse novo desenho há um desmembramento das duas grandes áreas disciplinares da saúde coletiva, isto é, epidemiologia/bioestatística e políticas e organização de serviços de saúde, cada uma com cargas horárias distintas, sendo a primeira nos $2^{\circ}$ e $3^{\circ}$ anos e a segunda no $4^{\circ}$ ano da graduação médica (situação que deve ser avaliada e, de alguma forma, comparada com a experiência anterior).

Antes dessa atual reforma, a saúde coletiva era uma disciplina anual, dividida em três módulos: epidemiologia, bioestatística e políticas e organização de serviços de saúde. Os temas da área de ciências sociais e humanas faziam parte do $1^{\circ}$ ano de Medicina, no módulo interdisciplinar Bases do Exercício Profissional (BEP), participando além da saúde coletiva, propedêutica e psicologia médica (módulo mantido no atual currículo).

Até aquele momento, um desafio que já se colocava para o processo de ensino-aprendizagem da saúde coletiva dizia respeito a sua inserção nos primeiros dois anos do curso médico, período de forte concentração de disciplinas de morfologia e fisiologia, em que os alunos têm aproximações incipientes com os serviços de saúde e baixa exposição dos problemas concretos de adoecimento e cuidado da população.

Apreender marcos teórico-metodológicos e desenvolver raciocínio descritivo e analítico sobre o fenômeno saúde-doença-cuidado são capacidades complexas que envolvem competências cognitivas e habilidades específicas. Há, além disso, as dimensões atitudinais das questões éticas e morais que o cuidado à saúde exige. Ensinar essas dimensões no momento em que a construção do saber morfofisiológico e patológico ainda está sendo processado, e sem uma inserção efetiva nas práticas de saúde, exige um enorme esforço de abstração, correndo o risco da disciplina ser demasiadamente teórica e muito "descolada" das atividades acadêmicas do aluno.

Essas questões trazidas pela inserção da saúde coletiva no curso médico e as preocupações com o ensinar, contribuindo para a construção de um pensamento crítico e mais próximo da realidade social sobre o fenômeno coletivo da saúde, doença e cuidado, fizeram com que estratégias pedagógicas fossem formuladas para superar esse enquadramento curricular, tais como: visitas aos serviços de saúde, pesquisa de assuntos cotidianos em mídias sobre saúde e sistemas de saúde, manejo de banco de dados do Ministério da Saúde, acompanhamentos de famílias no cuidado à saúde, seminários, convidados externos, estímulos à participação em eventos científicos da saúde coletiva, inserções nos projetos de extensão universitárias, como no PET-Saúde, trabalho de conclusão de disciplina, entre outros.

Uma dessas estratégias que obteve, em geral, bons resultados no processo de ensino-aprendizagem e que envolveu todos os docentes da disciplina, mesmo os que não estavam diretamente responsáveis pelos conteúdos programáticos, foi o trabalho de conclusão de disciplina.

Para essa estratégia, a turma (120 alunos) era dividida em pequenos grupos (mais ou menos, 6 alunos), sob orientação de um docente. Cada docente ficava com 3 a 4 grupos de alunos. Após uma exposição e problematização sobre o campo da saúde coletiva e os diferentes temas possíveis para estudar naquele momento, os grupos definiam os seus trabalhos, pactuados com os orientadores: doenças transmissíveis, doenças e agravos não transmissíveis, a questão cor na saúde, serviços de saúde mental, desigualdades sociais e saúde, urgência e emergência, atenção hospitalar, entre outros.

O período para desenvolver esse trabalho era o mesmo da disciplina, isto é, anual. No final do ano letivo, o grupo entregava o material escrito e o apresentava para uma "banca" de avaliadores externos (professores da Faculdade e/ou convidados de fora da Instituição que atuam na área em que o tema do grupo é desenvolvido).

Apesar de uma certa irregularidade no desenvolvimento de alguns desses trabalhos, imputada pelos alunos ao tempo destinado a essa tarefa no conjunto de outras exigências disciplinares, essa experiência foi, em geral, considerada positiva. Além do exercício teórico-metodológico de desenvolver temas de saúde coletiva, da própria apreensão de construção de projetos de pesquisa e de exercitar a produção intelectual e de uma certa "tutoria", muitos dos trabalhos foram apresentados nas sucessivas edições do Congresso Médico-Universitário do $\mathrm{ABC}$ e em Congressos da área de Saúde Coletiva. Alguns se transformaram em Projeto de Iniciação Científica.

Algumas dessas estratégias foram consolidadas e a outras não se deu continuidade. Entretanto, avaliamos que essas várias maneiras de exercitar e experimentar o processo de ensino da saúde coletiva na graduação médica foram, em geral, positivas. A pesquisa de Castellanos et al. ${ }^{12}$, que visou identificar nos egressos do curso médico da FMABC a avaliação sobre o ensino e a percepção a respeito da Atenção Primária à Saúde, revela que 24,3\% dos que responderam ao questionário consideram o que aprenderam com a saúde coletiva na Faculdade como excelente ou bom, principalmente no período de 1999-2004. Outro dado importante nessa 
direção é o resultado da prova do Conselho Regional de Medicina de São Paulo, que tem apresentado uma média de acerto alta nas questões de saúde pública, de acordo com os informes repassados para a Faculdade.

\section{DISCUSSÃO}

Pelo o que aqui se expôs, percebe-se a complexidade que envolve a dimensão do ensino da saúde coletiva na formação médica (que também podemos extrapolar para os demais cursos), muito em decorrência do seu próprio objeto, como também de ensinar para profissionais conteúdos que não fazem parte do seu núcleo técnico específico, num contexto de lógica hospitalocêntrica.

Esse é o desafio do saber e do saber fazer da docência, do ser professor, que o leva a pensar em possibilidades para superar os obstáculos à apreensão do conhecimento, visando, no caso específico da formação médica, aproximar o olhar crítico desses futuros profissionais das necessidades sociais e de saúde da população.

\section{REFERÊNCIAS}

1. Batista NA, Batista SH. Docência em saúde: temas e experiências. São Paulo: Senac São Paulo; 2004.

2. Schraiber LB. O médico e seu trabalho: limites da liberdade. São Paulo: Hucitec; 1993.

3. Costa NMSC. Docência no ensino médico: por que é tão difícil mudar? Rev Bras Educ Med. 2007;31(1):21-30. http://dx.doi.org/10.1590/S0100-55022007000100004

4. Feuerwerker L. Além do discurso de mudança na educação médica: processos e resultados. São Paulo: Hucitec; 2002.

5. Marsiglia RMG. Introdução. In: Marsiglia RMG. Ensino de graduação em saúde: ingresso e inserção profissional. FCMSCSP (1963-2013). São Paulo: Casa de Soluções; 2013; p.13-9.

6. Campos JJB, Elias PEM. A saúde coletiva no curso de medicina da Universidade Estadual de Londrina: reflexões iniciais. Rev Bras Educ Med. 2008;32(2):149-59. http://dx.doi.org/10.1590/S0100-55022008000200002

7. Oliveira JAA, Muniz Neto FJ, Pinto FJM, Silva MGC, Jorge MSB. A transversalidade do conhecimento da saúde coletiva no currículo de medicina de uma escola médica pública: relevância das disciplinas na formação dos alunos. Rev Bras Educ Med. 2010;34(2):278-83. http://dx.doi.org/10.1590/S0100-55022010000200012
8. Gomes LB, Sampaio J, Lins TS. Currículo de medicina na Universidade Federal da Paraíba: reflexões sobre uma experiência modular integrada com ênfase na atenção básica. Saúde Redes. 2015;1(1):39-46.

http://dx.doi.org/10.18310\%2F2446-4813.2015v1n1p39-46

9. Brasil. Constituição da República Federativa do Brasil. Titulo VIII da Ordem Social: Capítulo II Da Seguridade Social: Seção II da Saúde. Brasília: 1988.

10. Brasil. Conselho Nacional de Educação. Câmara de Educação Superior. Resolução CNE/CES n. 4, de 7 de novembro de 2001: Institui Diretrizes Curriculares Nacionais do Curso de Graduação em Medicina. Diário Oficial da União. Brasília: 2001.

11. Brasil. Ministério da Educação. Conselho Nacional de Educação. Câmara de Educação Superior. Resolução n.3, de 20 de junho de 2014: Institui Diretrizes Curriculares Nacionais do Curso de Graduação em Medicina e dá outras providências. Diário Oficial da União. Brasília: 2014.

12. Castellanos MEP, Silveira AFMH, Martins LC, Nascimento VB, Silva CS, Bortollotte FHB, et al. Perfil dos egressos da Faculdade de Medicina do ABC: o que eles pensam sobre atenção primária em saúde. Arq Bras Ciên Saúde. 2009;34(2):71-9. http://dx.doi.org/10.7322/abcs.v34i2.130 